\title{
NEW CLASSES OF HARMONIC UNIVALENT FUNCTIONS
}

\author{
G.Ş. SĂLĂGEAN, L.-I. COTîRLĂ
}

Abstract. We define and investigate new classes of harmonic univalent functions defined by Sălăgean integral operator, denoted by $H(m, n, \alpha, \beta)$ and $H^{-}(m, n, \alpha, \beta)$. We obtain coefficient inequalities and distortion bounds for the functions in the class $H(m, n, \alpha, \beta)$. We determine the extreme points of closed convex hulls of $H^{-}(m, n, \alpha, \beta)$, denoted by $\operatorname{clco} H^{-}(m, n, \alpha, \beta)$. We show that $H^{-}(m, n, \alpha, \beta)$ is closed under convex combination of its members.

2010 Mathematics Subject Classification: 30C45.

Keywords: Integral operator, harmonic univalent functions, distortion inequalities.

\section{INTRODUCTION}

A continuous complex valued function $f=u+i v$ defined in a complex domain $D$ is said to be harmonic in $D$ if both $u$ and $v$ are real harmonic in $D$. In any simply connected domain we can write $f=h+\bar{g}$, where $h$ and $g$ are analytic in $D$. A necessary and sufficient condition for $f$ to be locally univalent and sense preserving in $D$ is that $\left|h^{\prime}(z)\right|>\left|g^{\prime}(z)\right|, z \in D$.(See Clunie and Sheil-Small[2]).

Denote by $\mathcal{H}$ the class of functions $f=h+\bar{g}$ that are harmonic univalent and sense preserving in the unit $\operatorname{disc} U=\{z:|z|<1\}$ so that $f=h+\bar{g}$ is normalized by $f(0)=h(0)=f_{z}^{\prime}(0)-1=0$.

Let $\mathcal{H}(U)$ be the space of holomorphic functions in $U$. We let:

$$
A_{n}=\left\{f \in \mathcal{H}(U), f(z)=z+a_{n+1} z^{n+1}+\ldots, z \in U\right\}, \text { with } A_{1}=A .
$$

We let $\mathcal{H}[a, n]$ denote the class of analytic functions in $U$ of the form

$$
f(z)=a+a_{n} z^{n}+a_{n+1} z^{n+1}+\ldots, z \in U .
$$

The integral operator $I^{n}$ is defined in [4] by:

$$
(i) I^{0} f(z)=f(z)
$$




$$
\begin{gathered}
(i i) I^{1} f(z)=I f(z)=\int_{0}^{z} f(t) t^{-1} d t ; \\
(i i i) I^{n} f(z)=I\left(I^{n-1} f(z)\right), n \in \mathbb{N}-\{0\}, f \in A .
\end{gathered}
$$

Ahuja and Jahangiri [1] defined the class $H(n), n \in \mathbb{N}$, consisting of all univalent harmonic functions $f=h+\bar{g}$ that are sense preserving in $U$ and $h$ and $g$ are of the form:

$$
h(z)=z+\left\lceil\int \sum_{k=2}^{\infty} a_{k} z^{k}, g(z)=\left\lceil\int \sum_{k=1}^{\infty} b_{k} z^{k},\left|b_{1}\right|<1 .\right.\right.
$$

For $f=h+\bar{g}$ given by (1) the integral operator $I^{n}$ is defined as:

$$
I^{n} f(z)=I^{n} h(z)+(-1)^{n} \overline{I^{n} g(z)}, z \in U,
$$

where

$$
I^{n} h(z)=z+\left\lceil\int \sum_{k=2}^{\infty} k^{-n} a_{k} z^{k}\right.
$$

and

$$
I^{n} g(z)=\left\lceil\int \sum_{k=1}^{\infty} k^{-n} b_{k} z^{k} .\right.
$$

For fixed positive integers $n$ and for $0 \leq \alpha<1, \beta \geq 0, m \in \mathbb{N}, m \geq 1$, we let $H(m, n, \alpha, \beta)$ denote the class of univalent harmonic functions of the form (1) that satisfy the condition:

$$
\operatorname{Re}\left\{\frac{I^{n} f(z)}{I^{n+m} f(z)}\right\}>\beta\left|\frac{I^{n} f(z)}{I^{n+m} f(z)}-1\right|+\alpha .
$$

The subclass $H^{-}(m, n, \alpha, \beta)$ consists of functions $f_{n}=h+\overline{g_{n}}$ in $H(m, n, \alpha, \beta)$ so that $h$ and $g_{n}$ are of the form

$$
h(z)=z-\left\lceil\int \sum_{k=2}^{\infty} a_{k} z^{k}, g_{n}(z)=(-1)^{n-1}\left\lceil\int \sum_{k=1}^{\infty} b_{k} z^{k},\left|b_{1}\right|<1 .\right.\right.
$$

\section{THE MAIN RESULTS}

In the first theorem, we introduce a sufficient coefficient bound for harmonic functions in $H(m, n, \alpha, \beta)$. 
G.Ş. Sălăgean, L.-I. Cotîrlă - New classes of harmonic univalent functions

Theorem 1. Let $f=h+\bar{g}$ be given by (1). If

$$
\left\lceil\int \sum_{k=1}^{\infty}\left\{\psi(m, n, \alpha, \beta)\left|a_{k}\right|+\theta(m, n, \alpha, \beta)\left|b_{k}\right|\right\} \leq 2,\right.
$$

where

$$
\psi(m, n, \alpha, \beta)=\frac{k^{-n}(1+\beta)-(\beta+\alpha) k^{-(n+m)}}{1-\alpha},
$$

and

$$
\theta(m, n, \alpha, \beta)=\frac{k^{-n}(1+\beta)-(-1)^{m}(\beta+\alpha) k^{-(n+m)}}{1-\alpha},
$$

$a_{1}=1,0 \leq \alpha<1, \beta \geq 0, n \in \mathbb{N}, m \in \mathbb{N}, m \geq 1$, then $f \in H(m, n, \alpha, \beta)$.

Proof. According to (2) and(3) we only need to show that

$$
\operatorname{Re}\left(\frac{I^{n} f(z)-\alpha I^{n+m} f(z)-\beta e^{i \theta}\left|I^{n} f(z)-I^{n+m} f(z)\right|}{I^{n+m} f(z)}\right) \geq 0 .
$$

The case $r=0$ is obvious. For $0<r<1$ it follows that

$$
\begin{gathered}
\operatorname{Re}\left(\frac{I^{n} f(z)-\alpha I^{n+m} f(z)-\beta e^{i \theta}\left|I^{n} f(z)-I^{n+m} f(z)\right|}{I^{n+m} f(z)}\right)= \\
=\operatorname{Re}\left\{\frac{(1-\alpha) z+\left\lceil\int \sum_{k=2}^{\infty} a_{k} z^{k}\left[\gamma^{n}-\alpha \gamma^{n+m}\right]\right.}{z+\left\lceil\int \sum_{k=2}^{\infty} \gamma^{n+m} a_{k} z^{k}+(-1)^{n+m}\left\lceil\int \sum_{k=1}^{\infty} \gamma^{n+m} \overline{b_{k}} z^{k}\right.\right.}+\right. \\
+\frac{(-1)^{n}\left\lceil\int \sum_{k=1}^{\infty} \overline{b_{k}} \overline{z^{k}}\left[\gamma^{n}+\alpha \gamma^{n+m}\right]\right.}{z+\left\lceil\int \sum_{k=2}^{\infty} \gamma^{n+m} a_{k} z^{k}+(-1)^{n+m}\left\lceil\int \sum_{k=1}^{\infty} \gamma^{n+m} \overline{b_{k}} z^{k}\right.\right.}- \\
\left.-\frac{\beta e^{i \theta} \mid\left\lceil\int \sum_{k=2}^{\infty} a_{k} z^{k}\left[\gamma^{n}-\gamma^{n+m}\right]+(-1)^{n}\left\lceil\int \sum_{k=1}^{\infty} \overline{b_{k}} \overline{z^{k}}\left[\gamma^{n}+\gamma^{n+m}\right] \mid\right.\right.}{z+\left\lceil\int \sum_{k=2}^{\infty} \gamma^{n+m} a_{k} z^{k}+(-1)^{n+m}\left\lceil\int \sum_{k=1}^{\infty} \gamma^{n+m} \overline{b_{k}} \overline{z^{k}}\right.\right.}\right\}= \\
=\operatorname{Re}\left\{\frac{1-\alpha+\left\lceil\int \sum_{k=2}^{\infty} a_{k} z^{k-1}\left[\gamma^{n}-\alpha \gamma^{n+m}\right]\right.}{1+\left\lceil\int \sum_{k=2}^{\infty} \gamma^{n+m} a_{k} z^{k-1}+(-1)^{n+m}\left\lceil\int \sum_{k=1}^{\infty} \gamma^{n+m} \overline{b_{k}} \overline{z^{k}} z^{-1}\right.\right.}+\right. \\
\frac{(-1)^{n}\left\lceil\int \sum_{k=1}^{\infty} \overline{b_{k}} z^{k} z^{-1}\left[\gamma^{n}-(-1)^{m} \alpha \gamma^{n+m}\right]\right.}{1+\left\lceil\int \sum_{k=2}^{\infty} \gamma^{n+m} a_{k} z^{k-1}+(-1)^{n+m}\left\lceil\int \sum_{k=1}^{\infty} \gamma^{n+m} \overline{b_{k}} \overline{z^{k}} z^{-1}\right.\right.}- \\
\left.\frac{\beta e^{i \theta} z^{-1} \mid\left\lceil\int \sum_{k=2}^{\infty}\left[\gamma^{n}-\gamma^{n+m}\right] a_{k} z^{k}+(-1)^{n}\left\lceil\int \sum_{k=1}^{\infty}\left[\gamma^{n}-(-1)^{m} \gamma^{n+m}\right] \overline{b_{k}} \overline{z^{k}} \mid\right.\right.}{1+\left\lceil\int \sum_{k=2}^{\infty} \gamma^{n+m} a_{k} z^{k-1}+(-1)^{n+m}\left\lceil\int \sum_{k=1}^{\infty} \gamma^{n+m} \overline{b_{k}} \overline{z^{k}} z^{-1}\right.\right.}\right\}= \\
=R e \frac{(1-\alpha)+A(z)}{1+B(z)}, w h e r e \gamma=\frac{1}{k},
\end{gathered}
$$




$$
\begin{gathered}
A(z)=\left\lceil\int \sum_{k=2}^{\infty} a_{k} z^{k-1}\left[\gamma^{n}-\alpha \gamma^{n+m}\right]+(-1)^{n}\left\lceil\int \sum_{k=1}^{\infty} \overline{b_{k} z^{k}} z^{-1}\left[\gamma^{n}-(-1)^{m} \alpha \gamma^{n+m}\right]-\right.\right. \\
-\beta e^{i \theta} z^{-1} \mid\left\lceil\int \sum_{k=2}^{\infty}\left[\gamma^{n}-\gamma^{n+m}\right] a_{k} z^{k}+(-1)^{n}\left\lceil\int \sum_{k=1}^{\infty}\left(\gamma^{n}-(-1)^{m} \gamma^{n+m}\right) \overline{b_{k} z^{k}} \mid,\right.\right. \\
B(z)=\left\lceil\int \sum_{k=2}^{\infty} \gamma^{n+m} a_{k} z^{k-1}+(-1)^{n+m}\left\lceil\int \sum_{k=1}^{\infty} \gamma^{n+m} \overline{b_{k}} z^{k} z^{-1} .\right.\right.
\end{gathered}
$$

For $z=r e^{i \theta}$ we have

$$
\begin{gathered}
A\left(r e^{i \theta}\right)=\left\lceil\int \sum_{k=2}^{\infty}\left(\gamma^{n}-\alpha \gamma^{n+m}\right) a_{k} r^{k-1} e^{(k-1) \theta i}+\right. \\
+(-1)^{n}\left\lceil\int \sum_{k=1}^{\infty}\left(\gamma^{n}-(-1)^{m} \gamma^{n+m} \alpha\right) \overline{b_{k}} r^{k-1} e^{-(k+1) \theta i}-\beta \mathcal{D}(n+m, n, \alpha),\right.
\end{gathered}
$$

where

$$
\begin{gathered}
\mathcal{D}(n+m, n, \alpha)= \\
=\mid\left\lceil\int \sum_{k=2}^{\infty}\left(\gamma^{n}-\gamma^{n+m}\right) a_{k} r^{k-1} e^{-k i \theta}+(-1)^{n}\left\lceil\int \sum_{k=1}^{\infty}\left(\gamma^{n}-(-1)^{m} \gamma^{n+m}\right) \overline{b_{k}} r^{k-1} e^{-k i \theta} \mid,\right.\right.
\end{gathered}
$$

and

$$
B\left(r e^{i \theta}\right)=\left\lceil\int \sum_{k=2}^{\infty} \gamma^{n+m} a_{k} r^{k-1} e^{(k-1) \theta i}+(-1)^{n+m}\left\lceil\int \sum_{k=1}^{\infty} \gamma^{n+m} \overline{b_{k}} r^{k-1} e^{-(k+1) \theta i} .\right.\right.
$$

Setting $\frac{1-\alpha+A(z)}{1+B(z)}=(1-\alpha) \frac{1+w(z)}{1-w(z)}$.

The proof will be complete if we can show that $|w(z)| \leq r<1$. This is the case since, by the condition (5), we can write:

$$
\begin{gathered}
|w(z)|=\left|\frac{A(z)-(1-\alpha) B(z)}{A(z)+(1-\alpha) B(z)+2(1-\alpha)}\right| \leq \\
\frac{\left\lceil\int \sum_{k=1}^{\infty}\left[(1+\beta)\left(\gamma^{n}-\gamma^{n+m}\right)\left|a_{k}\right|+(1+\beta)\left(\gamma^{n}-(-1)^{m} \gamma^{n+m}\right)\left|b_{k}\right|\right] r^{k-1}\right.}{4(1-\alpha)-\left\lceil\int \sum_{k=1}^{\infty}\left\{\left[\gamma^{n}(1+\beta)-\delta \gamma^{n+m}\right]\left|a_{k}\right|+\left[\gamma^{n}(1+\beta)-(-1)^{m} \delta \gamma^{n+m}\right]\left|b_{k}\right|\right\} r^{k-1}\right.} \\
<\frac{\left\lceil\int \sum_{k=1}^{\infty}(1+\beta)\left(\gamma^{n}-\gamma^{n+m}\right)\left|a_{k}\right|+\left(\gamma^{n}-(-1)^{m} \gamma^{n+m}\right)(1+\beta)\left|b_{k}\right|\right.}{4(1-\alpha)-\left\lceil\int \sum_{k=1}^{\infty}\left\{\left[\gamma^{n}(1+\beta)-\delta \gamma^{n+m}\right]\left|a_{k}\right|+\left[\gamma^{n}(1+\beta)-(-1)^{m} \delta \gamma^{n+m}\right]\left|b_{k}\right|\right\}\right.} \\
\leq 1,
\end{gathered}
$$


G.Ş. Sălăgean, L.-I. Cotîrlă - New classes of harmonic univalent functions

where $\delta=\beta+2 \alpha-1$.

The harmonic univalent functions

$$
f(z)=z+\left\lceil\int \sum_{k=2}^{\infty} \frac{1}{\psi(m, n, \alpha, \beta)} x_{k} z^{k}+\left\lceil\int \sum_{k=1}^{\infty} \frac{1}{\theta(m, n, \alpha, \beta)} \overline{y_{k} z^{k}},\right.\right.
$$

where $n \in \mathbb{N}, 0 \leq \alpha<1, \beta \geq 0, m \in \mathbb{N}, m \geq 1$ and $\left\lceil\int \sum_{k=2}^{\infty}\left|x_{k}\right|+\left\lceil\int \sum_{k=1}^{\infty}\left|y_{k}\right|=1\right.\right.$, show that the coefficient bound given by (5) is sharp.

In the following theorem it is show that the condition (5)is also necessary for the function $f_{n}=h+\overline{g_{n}}$, where $h$ and $g_{n}$ are of the form (4).

Theorem 2. Let $f_{n}=h+\overline{g_{n}}$ be given by (4). Then $f_{n} \in H^{-}(m, n, \alpha, \beta)$ if and only if

$$
\begin{gathered}
\left\lceil\int \sum_{k=1}^{\infty}\left[\psi(m, n, \alpha, \beta) a_{k}+\theta(m, n, \alpha, \beta) b_{k}\right] \leq 2\right. \\
\quad a_{1}=1,0 \leq \alpha<1, n \in \mathbb{N}, m \in \mathbb{N}, m \geq 1
\end{gathered}
$$

Proof. Since $H^{-}(m, n, \alpha, \beta) \subset H(m, n, \alpha, \beta)$, we only need to prove the "only if" part of the theorem. For functions $f_{n}$ of the form (4), we note that the condition

$$
\operatorname{Re}\left\{\frac{I^{n} f(z)}{I^{n+m} f(z)}\right\}>\beta\left|\frac{I^{n} f(z)}{I^{n+m} f(z)}-1\right|+\alpha
$$

is equivalent to

$$
\begin{gathered}
\operatorname{Re}\left\{\frac{(1-\alpha) z-\left\lceil\int \sum_{k=2}^{\infty}\left(\gamma^{n}-\alpha \gamma^{n+m}\right) a_{k} z^{k}\right.}{z-\left\lceil\int \sum_{k=2}^{\infty} \gamma^{n+m} a_{k} z^{k}+(-1)^{2 n+m-1}\left\lceil\int \sum_{k=1}^{\infty} \gamma^{n+m} b_{k} \overline{z^{k}}\right.\right.}+\right. \\
+\frac{(-1)^{2 n-1}\left\lceil\int \sum_{k=1}^{\infty}\left(\gamma^{n}-(-1)^{m} \gamma^{n+m} \alpha\right) b_{k} \overline{z^{k}}\right.}{z-\left\lceil\int \sum_{k=2}^{\infty} \gamma^{n+m} a_{k} z^{k}+(-1)^{2 n+m-1}\left\lceil\int \sum_{k=1}^{\infty} \gamma^{n+m} b_{k} \overline{z^{k}}\right.\right.}- \\
\left.-\frac{\beta e^{i \theta} \mid-\left\lceil\int \sum_{k=2}^{\infty}\left(\gamma^{n}+\gamma^{n+m}\right) a_{k} z^{k}+(-1)^{2 n-1}\left\lceil\int \sum_{k=1}^{\infty}\left(\gamma^{n}-(-1)^{2 m} \gamma^{n+m}\right) \overline{b_{k}} \overline{z^{k}} \mid\right.\right.}{z-\left\lceil\int \sum_{k=2}^{\infty} \gamma^{n+m} a_{k} z^{k}+(-1)^{2 n+2 m-1}\left\lceil\int \sum_{k=1}^{\infty} \gamma^{n+m} b_{k} \overline{z^{k}}\right.\right.}\right\} \geq 0,
\end{gathered}
$$

where $\gamma=\frac{1}{k}$.

The above required condition (7) must hold for all values of $z \in U$. Upon choosing the values of $z$ on the positive real axis where $0 \leq z=r<1$, and using $\operatorname{Re}\left(-e^{i \theta}\right) \geq$ $-\left|e^{i \theta}\right|=-1$, we must have

$$
\frac{(1-\alpha)-\left\lceil\int \sum_{k=2}^{\infty}\left[\gamma^{n}(1+\beta)-(\alpha+\beta) \gamma^{n+m}\right] a_{k} r^{k-1}\right.}{1-\left\lceil\int \sum_{k=2}^{\infty} \gamma^{n+m} a_{k} r^{k-1}-(-1)^{m}\left\lceil\int \sum_{k=1}^{\infty} \gamma^{n+m} b_{k} r^{k-1}\right.\right.}-
$$




$$
-\frac{\left\lceil\int \sum_{k=1}^{\infty}\left[\gamma^{n}(1+\beta)+\gamma^{n+m}(\beta+\alpha)\right] b_{k} r^{k-1}\right.}{1-\left\lceil\int \sum_{k=2}^{\infty} \gamma^{n+m} a_{k} r^{k-1}-(-1)^{m}\left\lceil\int \sum_{k=1}^{\infty} \gamma^{n+m} b_{k} r^{k-1}\right.\right.} \geq 0 .
$$

If the condition (7) does not hold, then the expression in (8) is negative for $r$ sufficiently close to 1 . Hence there exist $z_{0}=r_{0}$ in $(0,1)$ for which this quotient in $(8)$ is negative. This contradicts the required condition for $f_{n} \in H^{-}(m, n, \alpha, \beta)$ and so the proof is complete.

The following theorem gives the distortion bounds for functions in $H^{-}(m, n, \alpha, \beta)$ which yields a covering results for this class.

Theorem 3. Let $f_{n} \in H^{-}(m, n, \alpha, \beta)$. Then for $|z|=r<1$ we have

$$
\left|f_{n}(z)\right| \leq\left(1+b_{1}\right) r+\left[\theta(m, n, \alpha, \beta)-\omega(m, n, \alpha, \beta) b_{1}\right] r^{n+m+1}
$$

and

$$
\left|f_{n}(z)\right| \geq\left(1-b_{1}\right) r-\left\{\phi(m, n, \alpha, \beta)-\omega(m, n, \alpha, \beta) b_{1}\right\} r^{n+m+1},
$$

where

$$
\begin{aligned}
\phi(m, n, \alpha, \beta) & =\frac{1-\alpha}{(1 / 2)^{n}(1+\beta)-(1 / 2)^{n+m}(\alpha+\beta)}, \\
\omega(m, n, \alpha, \beta) & =\frac{(1+\beta)-(-1)^{m}(\alpha+\beta)}{(1 / 2)^{n}(1+\beta)-(1 / 2)^{n+m}(\alpha+\beta)} .
\end{aligned}
$$

Proof. We prove the right side inequality for $\left|f_{n}\right|$. The proof for the left hand inequality can be done using similar arguments. Let $f_{n} \in H^{-}(m, n, \alpha, \beta)$. Taking the absolute value of $f_{n}$ then by Theorem 2 , we can obtain :

$$
\begin{gathered}
\left|f_{n}(z)\right|=\mid z-\left\lceil\int \sum_{k=2}^{\infty} a_{k} z^{k}+(-1)^{n-1}\left\lceil\int \sum_{k=1}^{\infty} b_{k} \overline{z^{k}} \mid \leq\right.\right. \\
\leq r+\left\lceil\int \sum_{k=2}^{\infty} a_{k} r^{k}+\left\lceil\int \sum_{k=1}^{\infty} b_{k} r^{k}=r+b_{1} r+\left\lceil\int \sum_{k=2}^{\infty}\left(a_{k}+b_{k}\right) r^{k} \leq\right.\right.\right. \\
\leq r+b_{1} r+\left\lceil\int \sum_{k=2}^{\infty}\left(a_{k}+b_{k}\right) r^{2}=\right. \\
=\left(1+b_{1}\right) r+\phi(m, n, \alpha, \beta)\left\lceil\int \sum_{k=2}^{\infty} \frac{1}{\phi(m, n, \alpha, \beta)}\left(a_{k}+b_{k}\right) r^{2} \leq\right. \\
\leq\left(1+b_{1}\right) r+\phi(m, n, \alpha, \beta) r^{n+m+1}\left\lceil\int \sum_{k=2}^{\infty}\left[\psi(m, n, \alpha, \beta) a_{k}+\theta(m, n, \alpha, \beta) b_{k}\right] \leq\right.
\end{gathered}
$$


G.Ş. Sălăgean, L.-I. Cotîrlă - New classes of harmonic univalent functions

$$
\leq\left(1+b_{1}\right) r+\left[\phi(m, n, \alpha, \beta)-\omega(m, n, \alpha, \beta) b_{1}\right] r^{n+m+1} \text {. }
$$

The following covering result follows from the left hand inequality in Theorem 3 .

Corollary 4. Let $f_{n} \in H^{-}(m, n, \alpha, \beta)$. Then for $|z|=r<1$ we have $\{w:|w|<$ $\left.1-b_{1}-\left[\phi(m, n, \alpha, \beta)-\omega(n, \alpha, \eta) b_{1}\right] \subset f_{n}(U)\right\}$.

Next we determine the extreme points of closed convex hulls of $H^{-}(m, n, \alpha, \beta)$, denoted by $\operatorname{clco} H^{-}(m, n, \alpha, \beta)$.

Theorem 5. Let $f_{n}$ be given by (4). Then $f_{n} \in H^{-}(m, n, \alpha, \beta)$ if and only if

$$
f_{n}(z)=\left\lceil\int \sum_{k=1}^{\infty}\left[x_{k} h_{k}(z)+y_{k} g_{n_{k}}(z)\right],\right.
$$

where $h(z)=z$,

$$
h_{k}(z)=z-\frac{1-\alpha}{k^{-n}(1+\beta)-(\beta+\alpha) k^{-(n+m)}} z^{k}, k=2,3, \ldots
$$

and

$$
g_{n_{k}}(z)=z+(-1)^{n-1} \frac{1-\alpha}{k^{-n}(1+\beta)-(-1)^{m}(\beta+\alpha) k^{-(n+m)}} \bar{z}^{k}, k=1,2,3, \ldots
$$

$x_{k} \geq 0, y_{k} \geq 0,\left\lceil\int \sum_{k=1}^{\infty}\left(x_{k}+y_{k}\right)=1\right.$.

In particular, the extreme points of $H^{-}(m, n, \alpha, \beta)$ are $\left\{h_{k}\right\}$ and $\left\{g_{n_{k}}\right\}$.

Proof. For functions $f_{n}$ of the form (5) we have:

$$
\begin{gathered}
f_{n}(z)=\left\lceil\int \sum_{k=2}^{\infty}\left[x_{k} h_{k}(z)+y_{k} g_{n_{k}}(z)\right]=\right. \\
=\left\lceil\int \sum_{k=1}^{\infty}\left(x_{k}+y_{k}\right) z-\left\lceil\int \sum_{k=2}^{\infty} \frac{1-\alpha}{k^{-n}(1+\beta)-(\beta+\alpha) k^{-(n+m)}} x_{k} z^{k}+\right.\right. \\
+(-1)^{n-1}\left\lceil\int \sum_{k=1}^{\infty} \frac{1-\alpha}{k^{-n}(1+\beta)-(-1)^{m}(\beta+\alpha) k^{-(n+m)}} y_{k} \bar{z}^{k} .\right.
\end{gathered}
$$


Then

$$
\begin{gathered}
\left\lceil\int \sum_{k=2}^{\infty} x_{k} \frac{k^{-n}(1+\beta)-(\beta+\alpha) k^{-(n+m)}}{1-\alpha} \cdot \frac{(1-\alpha)}{k^{-n}(1+\beta)-(\beta+\alpha) k^{-(n+m)}}+\right. \\
+\left\lceil\int \sum_{k=1}^{\infty} y_{k} \frac{k^{-n}(1+\beta)-(-1)^{m}(\beta+\alpha) k^{-(n+m)}}{1-\alpha} .\right. \\
\cdot \frac{1-\alpha}{k^{-n}(1+\beta)-(-1)^{m}(\beta+\alpha) k^{-(n+m)}} \\
=\left\lceil\int \sum_{k=2}^{\infty} x_{k}+\left\lceil\int \sum_{k=1}^{\infty} y_{k}=1-x_{1} \leq 1\right.\right.
\end{gathered}
$$

and so $f_{n}(z) \in H^{-}(m, n, \alpha, \beta)$.

Conversely, suppose $f_{n}(z) \in H^{-}(m, n, \alpha, \beta)$. Letting

$$
\begin{gathered}
x_{1}=1-\left\lceil\int \sum_{k=2}^{\infty} x_{k}-\left\lceil\int \sum_{k=1}^{\infty} y_{k}\right.\right. \\
x_{k}=\frac{k^{-n}(1+\beta)-(\beta+\alpha) k^{-(n+m)}}{1-\alpha} \cdot a_{k}, k=2,3, \ldots
\end{gathered}
$$

and

$$
y_{k}=\frac{k^{-n}(1+\beta)-(-1)^{m}(\beta+\alpha) k^{-(n+m)}}{1-\alpha} \cdot b_{k}, k=1,2,3, \ldots
$$

we obtain the required representation, since

$$
\begin{gathered}
f_{n}(z)=z-\left\lceil\int \sum_{k=2}^{\infty} a_{k} z^{k}+(-1)^{n-1}\left\lceil\int \sum_{k=1}^{\infty} b_{k} \bar{z}^{k}=\right.\right. \\
=z-\left\lceil\int \sum_{k=2}^{\infty} \frac{1-\alpha}{k^{-n}(1+\beta)-(\beta+\alpha) k^{-(n+m)}} x_{k} z^{k}+\right. \\
+(-1)^{n-1}\left\lceil\int \sum_{k=1}^{\infty} \frac{1-\alpha}{k^{-n}(1+\beta)-(-1)^{m}(\beta+\alpha) k^{-(n+m)}} y_{k} \bar{z}^{k}=\right. \\
=z-\left\lceil\int \sum_{k=2}^{\infty}\left[z-h_{k}(z)\right] x_{k}-\left\lceil\int \sum_{k=1}^{\infty}\left[z-g_{n_{k}}(z)\right] y_{k}=\right.\right. \\
=\left\lceil 1-\left\lceil\int \sum_{k=2}^{\infty} x_{k}-\left\lceil\int \sum_{k=1}^{\infty} y_{k}\right] z+\left\lceil\int \sum_{k=2}^{\infty} x_{k} h_{k}(z)+\left\lceil\int \sum_{k=1}^{\infty} y_{k} g_{n_{k}}(z)=\right.\right.\right.\right.
\end{gathered}
$$




$$
=\left\lceil\int \sum_{k=1}^{\infty}\left[x_{k} h_{k}(z)+y_{k} g_{n_{k}}(z)\right]\right.
$$

Now we show that $H^{-}(m, n, \alpha, \beta)$ is closed under convex combination of its members.

Theorem 6. The family $H^{-}(m, n, \alpha, \beta)$ is closed under convex combination.

Proof. For $i=1,2, \ldots$ suppose that $f_{n}^{i} \in H^{-}(m, n, \alpha, \beta)$, where

$$
f_{n}^{i}(z)=z+\left\lceil\int \sum_{k=2}^{\infty} a_{k}^{i} z^{k}+(-1)^{n-1}\left\lceil\int \sum_{k=1}^{\infty} b_{k}^{i} \bar{z}^{k},\right.\right.
$$

then by Theorem 2,

$$
\begin{gathered}
\left\lceil\int \sum_{k=1}^{\infty} \frac{k^{-n}(1+\beta)-(\beta+\alpha) k^{-(n+m)}}{1-\alpha} a_{k}^{i}+\right. \\
+\left\lceil\int \sum_{k=1}^{\infty} \frac{k^{-n}(1+\beta)-(-1)^{m}(\beta+\alpha) k^{-(n+m)}}{1-\alpha} b_{k}^{i} \leq 2,\right.
\end{gathered}
$$

for $\left\lceil\int \sum_{i=1}^{\infty} t_{i}=1,0 \leq t_{i} \leq 1\right.$, the convex combination of $f_{n}^{i}$ may be written as

$$
\left\lceil\int \sum_{i=1}^{\infty} t_{i} f_{n}^{i}(z)=z-\left\lceil\int \sum _ { k = 2 } ^ { \infty } \left(\left\lceil\int \sum_{i=1}^{\infty} t_{i} a_{k}^{i}\right) z^{k}+(-1)^{n-1}\left\lceil\int \sum _ { k = 1 } ^ { \infty } \left(\left\lceil\int \sum_{i=1}^{\infty} t_{i} b_{k}^{i}\right) \bar{z}^{k} .\right.\right.\right.\right.\right.
$$

Then by (8)

$$
\begin{gathered}
\left\lceil\int \sum _ { k = 1 } ^ { \infty } \frac { k ^ { - n } ( 1 + \beta ) - ( \beta + \alpha ) k ^ { - ( n + m ) } } { 1 - \alpha } \left(\left\lceil\int \sum_{i=1}^{\infty} t_{i} a_{k}^{i}\right)+\right.\right. \\
+\left\lceil\int \sum _ { k = 1 } ^ { \infty } \frac { k ^ { - n } ( 1 + \beta ) - ( - 1 ) ^ { m } ( \beta + \alpha ) k ^ { - ( n + m ) } } { 1 - \alpha } \left(\left\lceil\int \sum_{i=1}^{\infty} t_{i} b_{k}^{i}\right)=\right.\right. \\
=\left\lceil\int \sum _ { i = 1 } ^ { \infty } t _ { i } \left[\left\lceil\int \sum_{k=1}^{\infty} \frac{k^{-n}(1+\beta)-(\beta+\alpha) k^{-(n+m)}}{1-\alpha} a_{k}^{i}+\right.\right.\right. \\
+\left\lceil\int \sum_{k=1}^{\infty} \frac{k^{-n}(1+\beta)-(-1)^{m}(\beta+\alpha) k^{-(n+m)}}{1-\alpha} b_{k}^{i}\right] \\
\leq 2\left\lceil\int \sum_{i=1}^{\infty} t_{i}=2\right.
\end{gathered}
$$

and therefore $\left\lceil\int \sum_{i=1}^{\infty} t_{i} f_{n}^{i}(z) \in H^{-}(m, n, \alpha, \beta)\right.$. 
G.Ş. Sălăgean, L.-I. Cotîrlă - New classes of harmonic univalent functions

Acknowledgements. The beautiful results for harmonic functions, was obtained by P. T. Mocanu in [3].

\section{REFERENCES}

[1] O.P. Ahuja, J.M. Jahangiri, Multivalent harmonic starlike functions, Ann. Univ. Marie Curie-Sklodowska Sect. A, LV 1(2001), 1-13.

[2] J. Clunie, T. Scheil- Small, Harmonic univalent functions, Ann. Acad. Sci. Fenn. Ser. A. I. Math., 9(1984), 3-25.

[3] P. T. Mocanu, Three-cornered hat harmonic functions, Complex Variables and Elliptic Equation, 12(2009), 1079-1084.

[4] G.S. Sălăgean, Subclass of univalent functions, Lecture Notes in Math. SpringerVerlag, 1013(1983), 362-372.

Grigore Ştefan Sălăgean

Babeş Bolyai University

Faculty of Mathematics and Computer Science

Cluj Napoca, Romania

email: salagean@math.ubbcluj.ro

Luminita-Ioana Cotîrlă

Technical University

Department of Mathematics

Cluj-Napoca, Romania

email: Luminita.Cotirla@math.utcluj.ro 